\title{
Commentary
}

\section{Finding Outbreaks Faster}

Mark S. Smolinski, Adam W. Crawley, and Jennifer M. Olsen

Rapid detection, reporting, and response to an infectious disease outbreak are critical to prevent localized health events from emerging as pandemic threats. Metrics to evaluate the timeliness of these critical activities, however, are lacking. Easily understood and comparable measures for tracking progress and encouraging investment in rapid detection, reporting, and response are sorely needed. We propose that the timeliness of outbreak detection, reporting, laboratory confirmation, response, and public communication should be considered as measures for improving global health security at the national level, allowing countries to track progress over time and inform investments in disease surveillance.

$\mathbf{R}$ APIDLY DETECTING, REPORTING, AND RESPONDING to infectious disease emergence is required to contain small outbreaks before they have the opportunity to spread into a regional epidemic or become a global pandemic threat. Rapid detection depends on effective disease surveillance systems leveraging data from multiple sources. Timeliness is a key criterion for evaluating any disease surveillance system. How fast a system can detect a threat is critical for ensuring optimal performance.

Continuously evaluating timeliness can help monitor improvements in the system over time. Of course, in determining whether a surveillance system is "timely," multiple factors must be considered, including disease transmissibility, incubation period, duration of infectiousness, severity of outcomes, and the objectives of the surveillance system in question. Despite this complexity, we believe a set of standardized metrics is plausible, if not essential, for monitoring progress toward improved disease surveillance. Few studies have evaluated infectious disease surveillance systems through the lens of the time required to identify and report an outbreak or unusual disease occurrence. Among the studies that do exist, standardization is lacking. ${ }^{1-5}$

The Ebola epidemic in West Africa in 2014 and the rapid emergence of Zika virus in South America in 2015 have brought increased attention to the risk that growing epidemics pose to global health security. ${ }^{6}$ Metrics to evaluate global health security, however, remain elusive. Easily understood and comparable measures for tracking progress and encouraging investment in rapid detection, reporting, and response are sorely needed. We propose that the timeliness of outbreak detection, reporting, laboratory confirmation, response, and appropriate public communication should be considered as one set of measures for global health security.

Mark S. Smolinski, MD, MPH, is Chief Medical Officer and Director, Global Health Threats; Adam W. Crawley, MPH, is a Research Associate, Pandemics; and Jennifer M. Olsen, DrPH, is Manager, Pandemics; all are at the Skoll Global Threats Fund, San Francisco, California.

(C) Mark S. Smolinski et al., 2017; Published by Mary Ann Liebert, Inc. This Open Access article is distributed under the terms of the Creative Commons Attribution Noncommercial License (http://creativecommons.org/licenses/by-nc/4.0/) which permits any noncommercial use, distribution, and reproduction in any medium, provided the original author(s) and the source are credited. 


\section{IHR AND GHSA}

To ensure national and global health security, finding, reporting, and controlling every outbreak faster, no matter where it happens around the globe, is paramount. The world has recognized this for some time. In 2005, the World Health Organization (WHO) revised the International Health Regulations (IHR) to create a better framework for disease reporting. ${ }^{7}$ These revisions broadened the scope of the IHR beyond reporting a handful of select diseases, instead focusing on identifying any public health emergency of international concern (PHEIC). A significant change in the new IHR permitted the WHO to act on "unofficial" information, such as outbreak reports obtained through digital disease detection systems like ProMEDmail and the Global Public Health Intelligence Network (GPHIN). The 2005 IHR charged every country to develop the capacity to detect outbreaks within its borders and to report them in a timely manner. It has often been noted that a progress report to $\mathrm{WHO}$ in 2012 revealed that approximately $80 \%$ of the 194 states parties had failed to meet their obligations under the IHR or simply did not report on their progress. ${ }^{8}$ Since that time, progress toward IHR compliance has continued, but many countries remain well short of their obligations. ${ }^{9}$

In a complementary effort to the IHR, the Global Health Security Agenda (GHSA) was established in February 2014. The GHSA was created to "accelerate progress toward a world safe and secure from infectious disease threats and to promote global health security as an international security priority." ${ }^{10}$ By 2016 more than 50 countries and international partners, including the WHO, the World Organization for Animal Health (OIE), and the Food and Agriculture Organization of the United Nations (FAO) had signed on to the GHSA. A main focus of the GHSA is to support countries working toward IHR compliance. ${ }^{11}$

The IHR and the GHSA both provide useful frameworks and engage the international community in jointly addressing emerging infectious disease threats. Clear performance measures, however, are still needed to track progress under either framework. The IHR Core Capacities, particularly Core Capacity 3: Surveillance, emphasize the importance of early detection. The recommended IHR checklist for monitoring progress does so only through measuring capability levels on a 4 -point scale of $<1$ to $3 .^{12}$ To improve on existing metrics, WHO convened a technical consultation in October 2015 on monitoring and evaluating IHR implementation and compliance that laid the groundwork for a new post-2016 IHR monitoring and evaluation framework. ${ }^{13}$ The Joint External Evaluation (JEE) framework that has been established in coordination with the GHSA provides a useful peer-to-peer evaluation approach, but it remains to be seen how shortfalls identified by the JEE process will be monitored for improvement beyond the assessment period. ${ }^{14}$
Areas at highest risk for emerging diseases may benefit most from tracking timeliness measures, but all countries working toward (and maintaining) IHR compliance will benefit from the insight that these measures can bring. Global health security carries with it a mandate to find every outbreak of pandemic potential, and we must ensure that all communities have the capacity to rapidly report all communicable disease events and clusters of unusual illness. The faster we can detect any suspected outbreak and implement rapid response, the more likely it is that we will avoid a repeat of the Ebola outbreak in West Africa that killed more than 11,000 victims and persisted long enough to devastate the already weak healthcare systems in affected countries.

We need clear goals and incentives to increase our global capacity for finding and reporting outbreaks faster to save lives, protect livelihoods, and ensure the risk of a pandemic is removed from our collective pool of worry. We should make finding and reporting every outbreak faster the central goal of pandemic preparedness. We cannot rely on pandemic preparedness plans, which often sit on the shelf in very large binders, to be implemented seamlessly during an emergency. Rather, pandemic preparedness should be seen as the active process of measuring and assessing progress in these timeliness measures that may ensure more effective improvements in surveillance. We can build disease surveillance systems that help every country meet the IHR while also addressing local needs based on risk and burden of disease by enhancing the capacity to detect aberrant signals as an early warning of any emerging threat.

\section{Methods for Quantifying Timeliness MeAsures}

We propose that the timeliness of outbreak detection, reporting, laboratory confirmation, response, and appropriate public communication could be adopted as key measures for monitoring pandemic preparedness and global health security. These metrics can be useful to ministries of health, along with other local, regional, and global partners in disease prevention and control. If these data were tracked for every outbreak, the results could be used to improve performance at the village, district, and provincial levels in every country. Nations could use these metrics to measure progress toward meeting the IHR or achieving the intent of the GHSA. Regional disease surveillance networks, such as those operating under the Connecting Organizations for Regional Disease Surveillance (CORDS) umbrella, could monitor progress as a cohesive unit, helping each other to constantly seek improvement and to identify best practices. ${ }^{15}$

The timeliness measures, illustrated in Table 1, involve quantifying the time interval between disease onset in the index case of a given outbreak and (a) the date that the 
Table 1. Working Definitions for Outbreak Milestones

\begin{tabular}{|c|c|}
\hline Outbreak Milestone & Definition \\
\hline Date of outbreak start & $\begin{array}{l}\text { Date of the symptoms onset of the index case. If it is not available, first date of hospitalization or medical } \\
\text { visit may be used. }\end{array}$ \\
\hline $\begin{array}{l}\text { Date of outbreak } \\
\text { detection }\end{array}$ & $\begin{array}{l}\text { Date that the outbreak threshold }{ }^{\text {a }} \text { case presents to a hospital, clinic, laboratory, community health } \\
\text { worker, or public health agency. }\end{array}$ \\
\hline $\begin{array}{l}\text { Date of outbreak } \\
\text { reporting }\end{array}$ & $\begin{array}{l}\text { Date that the outbreak threshold case is reported to public health authorities at local, regional, national, } \\
\text { or international level. }\end{array}$ \\
\hline $\begin{array}{l}\text { Date of laboratory } \\
\text { confirmation }\end{array}$ & Date of the first laboratory report of the causative pathogen from an epidemiologically linked case. \\
\hline $\begin{array}{l}\text { Date of public health } \\
\text { response }\end{array}$ & $\begin{array}{l}\text { Earliest date when the local public health professionals took actions to stop or control the outbreak in the } \\
\text { community. }\end{array}$ \\
\hline $\begin{array}{l}\text { Date of first public } \\
\text { communication }\end{array}$ & $\begin{array}{l}\text { Date that public information about the outbreak appeared in local or international, informal or official, } \\
\text { verbal or written reports, including the official press releases or newspapers in the country, news } \\
\text { articles, TV or radio broadcasts, internet postings, social media, or informal disease reporting networks } \\
\text { such as ProMED or HealthMap. }\end{array}$ \\
\hline
\end{tabular}

${ }^{a}$ Outbreak threshold refers to minimum number of cases and other criteria required to declare an outbreak for a particular disease.

outbreak was detected,* (b) the date the outbreak was reported to health authorities, (c) the date of laboratory confirmation of the causative pathogen from an epidemiologically linked case, and (d) the date of implementation of initial control measures. The date of communication about the outbreak to relevant national or international authorities may also be considered for evaluation. Working with our partner organizations across the globe, the Skoll Global Threats Fund (SGTF) is enabling active tracking of timeliness metrics at a national level, adapted from an existing methodology. ${ }^{16,17}$

In 2010 Chan et al examined the timelines for 281 WHO-verified outbreaks reported in Disease Outbreak News from 1996 to $2009 .{ }^{16}$ The study found that the median time from "outbreak start" to "outbreak discovery" dropped from 40 days to 19 days during that time. The study also examined other outbreak "milestones," including laboratory confirmation and public communication about the outbreak. ${ }^{16}$ A subsequent 5 -year update to the original study found that little progress has been made since 2010. ${ }^{17}$

Establishing a baseline for timeliness of outbreak detection on a global scale provides a broad view of the timeliness of outbreak surveillance capabilities around the world. The small number of observations in the Disease Outbreak News dataset, however, limits our ability to examine trends by country or disease. As the basic reproductive number $\left(r_{0}\right)$, incubation period, mode of transmission, treatment, and vaccination options for different pathogens vary considerably, 19 days until an outbreak is detected may allow for significant spread in one instance or may result in relatively

*The minimum number of cases to reach the threshold for an outbreak is determined based on several factors, including the cause of the disease and other factors predetermined by each country. few secondary cases in another, depending on the disease, locale, and other factors. It is therefore critical to obtain more granular data on the national and subnational levels, stratified by disease type, to better understand how each country's surveillance systems are performing against the demands to find, report, and respond to outbreaks faster.

In 2014 representatives from HealthMap of Boston Children's Hospital, the International Society for Infectious Diseases' Program for Monitoring Emerging Diseases (ProMED-mail), the Task Force for Global Health's Training Programs in Epidemiology and Public Health Interventions (TEPHINET), and SGTF convened to adapt the methods used in Chan et al for use at the national level. Through TEPHINET - the umbrella organization for 55 field epidemiology training programs (FETPs) around the world, the majority of which receive CDC support-field epidemiology training programs were encouraged to submit applications for grant funding to support the implementation of these methods in their own country. ${ }^{18}$ At the time of this writing, SGTF has worked with TEPHINET to provide support to field epidemiology training programs and their associated ministries of health in 17 countries as they examine their detection, reporting, verification, response, and communication timeliness for infectious disease outbreaks. Through 3 rounds of funding, awardees from Barbados, Belize, Costa Rica, Dominican Republic, Guatemala, Haiti, Honduras, India, Indonesia, Kenya, Pakistan, Panama, Paraguay, Senegal, Taiwan, Tanzania, and Zimbabwe have been selected to carry out evaluative studies (Figure 1). In addition, SGTF is working with the Mekong Basin Disease Surveillance network (MBDS) and Mahidol University in Bangkok, Thailand, to conduct a similar study on a regional level that engages Cambodia, Laos, Myanmar, and Vietnam. Plans to replicate this work in southeast Europe through partnership with the Southeast European Center for 


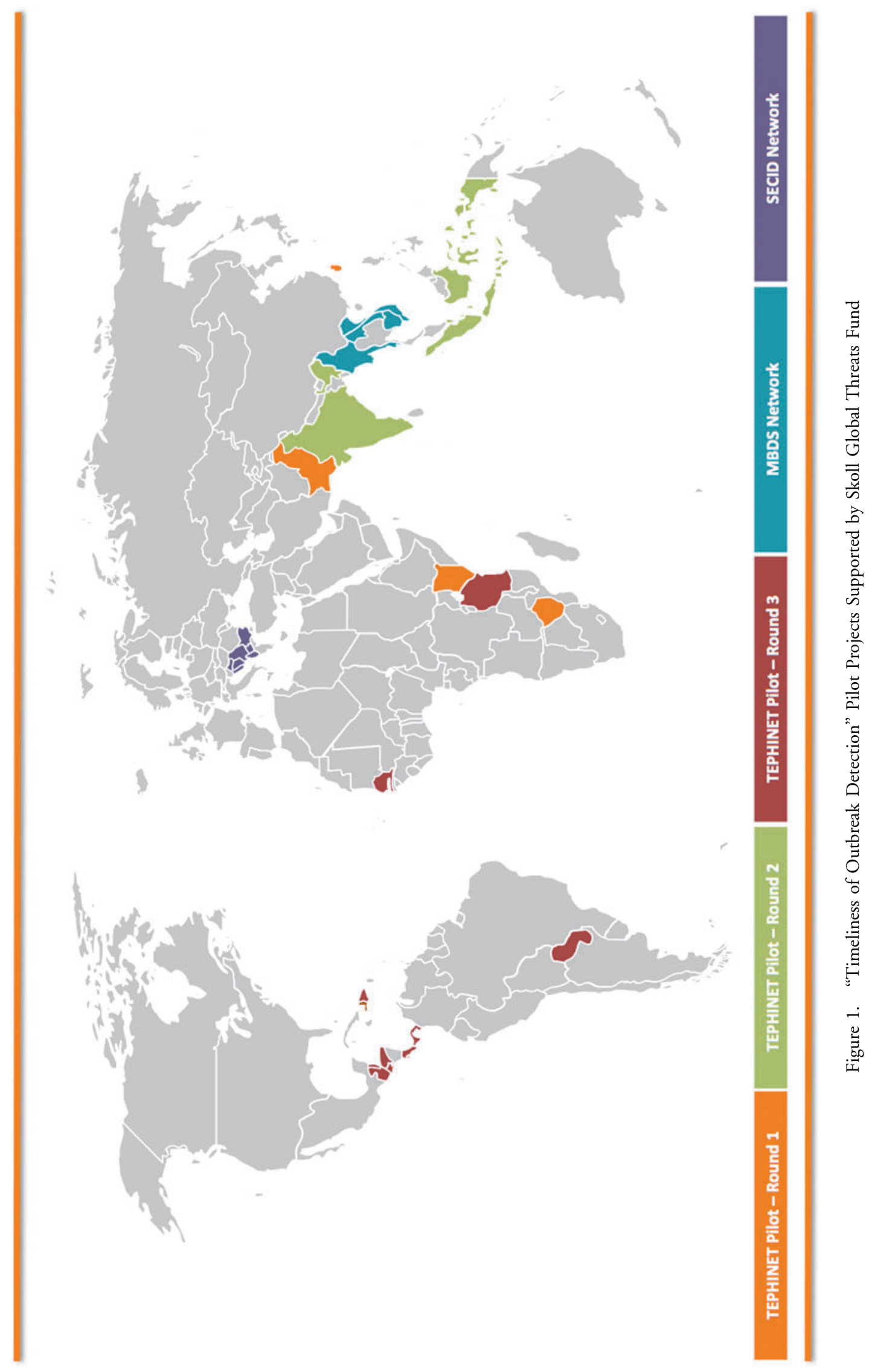


Surveillance and Control of Infectious Diseases (SECID) are also in place. As results from these pilot studies are made available throughout 2017, we hope to engage key stakeholders in the global health security community in the process of refining these measures and helping to implement them in additional countries.

In addition to examining data from their own national surveillance systems, the pilot countries are reviewing the timelines of outbreak reports from informal, event-based surveillance systems, including, but not limited to, HealthMap, ProMED-mail, and GPHIN. By examining data from these informal systems, public health authorities can also use this methodology to assess how emerging digital disease detection systems may complement traditional surveillance, especially in regions with limited surveillance infrastructure and resources.

\section{Challenges and Limitations}

Even with clear and precise definitions for each of these outbreak metrics, obtaining the relevant data points remains challenging. Determining the date of onset in an index case can require significant investigative work and, for many outbreaks, may never be known. How the overall timeline for an outbreak should be measured is subject to questions such as whether measurement starts during symptom onset in the index case, or perhaps when the $n$th case in an outbreak is presented and reaches the minimum number of cases to meet an outbreak definition - a definition that may vary by country and by disease.

Additionally, countries working to establish baseline data for these measures may not have all relevant data points captured in historical records, or the process of data extraction may require significant effort. For example, we have found through our pilot studies in Southeast Asia that the time of outbreak detection (as defined in this article) is often unavailable, but the time of reporting, arguably the more relevant data point, is typically obtainable. Finally, while evidence reveals that faster detection and response to infectious disease outbreaks help to reduce the overall number of cases, it remains to be seen whether process improvements that may be reflected in these metrics would translate to improving health outcomes in a population. ${ }^{19,20}$

Despite these challenges, we are confident that nations undertaking efforts to establish a baseline for outbreak timeliness metrics through retrospective evaluation will be able to learn whether changes to reporting policies, use of novel disease reporting systems, and/or increased investments in disease surveillance improve the speed of detection, reporting, and response. Ultimately, we hope to demonstrate that more timely performance results in reduced morbidity and mortality. We hope that timeliness metrics can identify programmatic or geographic gaps to inform resource allocation and strategic planning. Governments, NGOs, and philanthropies, working in part- nership, will be able to better understand which investments have the biggest impact in the area of outbreak surveillance and can share this knowledge to ensure efficient use of limited resources.

Measuring the time to detect, report, verify, respond, and communicate can help track progress toward finding and reporting outbreaks faster and move each country toward improved national and global health security. We need standardized metrics for monitoring progress, and we hope our investments in timeliness metrics for national and global health security will help refine our methods and allow for a rigorous approach that all nations can eventually adopt. We will continue to work with individual nations and regional networks to refine our approach and look forward to sharing our collective knowledge with the global health community.

\section{ACKNOWLEDGMENTS}

The authors acknowledge the authors of Chan et al (2010) for their work in developing an initial approach to capturing these metrics and the authors of Kluberg et al (2016) for their work in updating the original study. We would specifically like to thank Larry Madoff (ProMED-mail), Sumiko Mekaru (HealthMap), Sheryl Kluberg (HealthMap), Anika Vinze (TEPHINET), and Marlo Libel (Global Health LLC) for their contributions to developing the methodology for use by field epidemiology training programs at the national level. We would also like to thank TEPHINET for their role in supporting pilot studies of this approach, as well as the field epidemiologists implementing the studies. Finally, we would like to acknowledge the Mekong Basin Disease Surveillance network and the Southeast European Center for Surveillance and Control of Infectious Diseases for working with us to implement this approach in their respective regions.

\section{REFERENCES}

1. Reijn E, Swaan CM, Kretzschmar ME, van Steenbergen JE. Analysis of timeliness of infectious disease reporting in the Netherlands. BMC Public Health 2011;11:409.

2. Jajosky RA, Groseclose SL. Evaluation of reporting timeliness of public health surveillance systems for infectious diseases. BMC Public Health 2004;4:29.

3. Mondor L, Brownstein JS, Chan E, et al. Timeliness of nongovernmental versus governmental global outbreak communications. Emerg Infect Dis 2012;18(7):1184-1187.

4. Tsai FJ, Tseng E, Chan CC, Tamashiro H, Motamed S, Rougemont AC. Is the reporting timeliness gap for avian flu and $\mathrm{H} 1 \mathrm{~N} 1$ outbreaks in global health surveillance systems associated with country transparency? Global Health 2013;9:14.

5. Bahk CY, Scales DA, Mekaru SR, Brownstein JS, Freifeld CC. Comparing timeliness, content, and disease severity of formal and informal source outbreak reporting. BMC Infect Dis 2015;15(1):135. 


\section{FINDING OUTBREAKS FASTER}

6. Faria NR, Azevedo RS, Kraemer MU, et al. Zika virus in the Americas: early epidemiological and genetic findings. Science 2016;352(6283):345-349.

7. World Health Organization. International Health Regulations (2005). http://www.who.int/ihr/publications/9789241596664/ en/index.html. Accessed February 22, 2017.

8. World Health Organization. Implementation of the International Health Regulations (2005). Report by the DirectorGeneral. 2013. http://apps.who.int/gb/ebwha/pdf_files/ WHA66/A66_16-en.pdf. Accessed February 22, 2017.

9. World Health Organization. Global Health Observatory data repository. Surveillance. Data by country. Updated April 12, 2014. http://apps.who.int/gho/data/view.main. IHRCTRY03v?lang=en. Accessed February 22, 2017.

10. Global Health Security Agenda. https://www.ghsagenda.org/. Accessed February 22, 2017.

11. Inglesby T, Fischer JE. Moving ahead on the Global Health Security Agenda. Biosecur Bioterror 2014;12(2):63-65.

12. World Health Organization. Checklist and Indicators for Monitoring Progress in the Development of IHR Core Capacities in States Parties. 2013. http://www.who.int/ihr/checklist/en/. Accessed February 22, 2017.

13. World Health Organization. Technical Consultation on Monitoring and Evaluation of Functional Core Capacity for Implementing the International Health Regulations (2005). 2015. http://www.who.int/ihr/publications/WHO_HSE_ GCR_2015.14/en/. Accessed February 22, 2017.

14. World Health Organization. Joint External Evaluation Tool: International Health Regulations (2005). 2016. http://www. who.int/iris/handle/10665/204368. Accessed February 22, 2017.

15. Gresham LS, Smolinski MS, Suphanchaimat R, Kimball AM, Wibulpolprasert S. Creating a global dialogue on infectious disease surveillance: Connecting Organizations for
Regional Disease Surveillance (CORDS). Emerg Health Threats J 2013;6.

16. Chan EH, Brewer TF, Madoff LC, et al. Global capacity for emerging infectious disease detection. Proc Natl Acad Sci US A 2010;107(50):21701-21706.

17. Kluberg SA, Mekaru SR, McIver DJ, et al. Global capacity for emerging infectious disease detection, 1996-2014. Emerg Infect Dis 2016;22(10):E1-6.

18. Centers for Disease Control and Prevention. Global Health Protection and Security. Field epidemiology training program (FETP). Updated May 9, 2016. http://www.cdc.gov/ globalhealth/fetp/. Accessed February 22, 2017.

19. Zhang Y, Lopez-Gatell H, Alpuche-Aranda CM, Stoto MA. Did advances in global surveillance and notification systems make a difference in the $2009 \mathrm{H} 1 \mathrm{~N} 1$ pandemic?-a retrospective analysis. PLoS One 2013;8(4):e59893.

20. Blake IM, Chenoweth P, Okayasu H, Donnelly C, Aylward RB, Grassly NC. Faster detection of poliomyelitis outbreaks to support polio eradication. Emerg Infect Dis 2016;22(3): 449-456.

Manuscript received August 15, 2016;

revision returned December 19, 2016;

accepted for publication December 22, 2016.

Address correspondence to: Adam W. Crawley Skoll Global Threats Fund Pandemics

1808 Wedemeyer St., Suite 300 San Francisco, CA 94129

E-mail: acrawley@skollglobalthreats.org 